Anim. Reprod., v.16, n.1, p.45-51, Jan./Mar. 2019

\title{
Bioengineering the ovary to preserve and reestablish female fertility
}

\author{
Fulvio Gandolfi ${ }^{1 \S}$, Matteo Ghiringhelli ${ }^{2}$, Tiziana A.L. Brevini ${ }^{2}$ \\ ${ }^{1}$ Department of Agricultural and Environmental Sciences - Production, Landscape, Agroenergy, Università degli Studi di Milano, \\ Milano 20122, Italy. \\ ${ }^{2}$ Department of Health, Animal Science and Food Safety, Università degli Studi di Milano, Milano 20122, Italy.
}

\begin{abstract}
Different bioengineering strategies can be presently adopted and have been shown to have great potential in the treatment of female infertility and ovarian dysfunction deriving from chemotherapy, congenital malformations, massive adhesions as well as aging and lifestyle. One option is transplantation of fresh or cryopreserved organs/fragments into the patient. A further possibility uses tissue engineering approaches that involve a combination of cells, biomaterials and factors that stimulate local ability to regenerate/ repair the reproductive organ. Organ transplant has shown promising results in large animal models. However, the source of the organ needs to be identified and the immunogenic effects of allografts remain still to be solved before the technology may enter the clinical practice. Decellularization/ repopulation of ovary with autologous cells or follicles could represent an interesting, still very experimental alternative. Here we summarize the recent advancements in the bioengineering strategies applied to the ovary, we present the principles for these systems and discuss the advantages of these emerging opportunities to preserve or improve female fertility.
\end{abstract}

Keywords: cryopreservation, decellularization, female infertility, ovary, tissue engineering, 3D printing.

\section{Introduction}

Ovary dysfunction and failure is one of the main causes for infertility, with an alarming incidence of one out of 1000 women, under the age of 30 , rising to one $\%$ in women before 40 (Hewlett and Mahalingaiah, 2015; Qin et al., 2015). Patients affected are not able to undergo physiological cycles and/or release oocytes nor they produce normal levels of hormones. Several potential causes have been described. Radio-therapy and/or chemotherapy, viral infections, environmental factors, metabolic and autoimmune diseases, and genetic alterations (Yalcinkaya et al., 2014; SadriArdekani and Atala, 2015; Qin et al., 2015). Studies performed in the last years have shown for instance that the use of alkylating agents associated with abdominal ionizing radiation will render infertile almost $100 \%$ of the patients prepared for bone marrow transplantation (Wallace et al., 2005; Anderson and Wallace, 2011; Donnez et al., 2013). In addition, the probability of normal full term pregnancy is reduced to $30 \%-50 \%$ in cancer survivors compared to the corresponding healthy general population (Chung et al., 2013).

Several options to restore ovarian function have been developed in the last years and are under assessment at present. These include preservation and transplantation of autologous ovarian fragments and/or whole ovaries into the patient, with no issues related to rejection or need for immunosuppression. However, since this procedure is largely devoted to cancer patients, there is the real danger that the ovarian tissue which is transplanted back may contains malignant cells, posing a severe risk to the patient (Meirow et al., 2008; Dolmans et al., 2010; Greve et al., 2012).

Tissue engineered ovarian scaffold and ovarian culture systems have been proposed as an alternative. This approach has been mainly designed to avoid the possibility to reintroduce the disease. It involves the use of 3D in vitro culture system, based on polymers of different sort and alginate and has allowed for the creation of artificial ovary scaffolds in vitro (Pangas et $a l, 2003$; Xu et al, 2006). However most of the data available are limited to rodents. Furthermore, the biomaterials used alone lacked extracellular matrix (ECM) proteins that are essential to the phenotypes of mammalian cells (Huet et al., 2001; Che-Ying et al., 2016; Krotz et al., 2010). Because of this, ECM components such as arginine-glycine-aspartate (RGD), collagen (type I and IV), and/or fibronectin have been added to the matrix and led to improved growth, differentiation, and meiotic competence of the oocyte (Kreeger et al., 2003; Kreeger et al, 2006; Laronda et al., 2015). An experimental option is presently represented by the creation of biological scaffolds that are obtained through a decellularization process that removes all the original resident cells but leaves the ECM intact. This matrix is then repopulated with the patient cells, and can finally be transplanted. This protocol has been recently described and shown to successfully initiate puberty in mice (Laronda et al., 2015; Fig.1). 


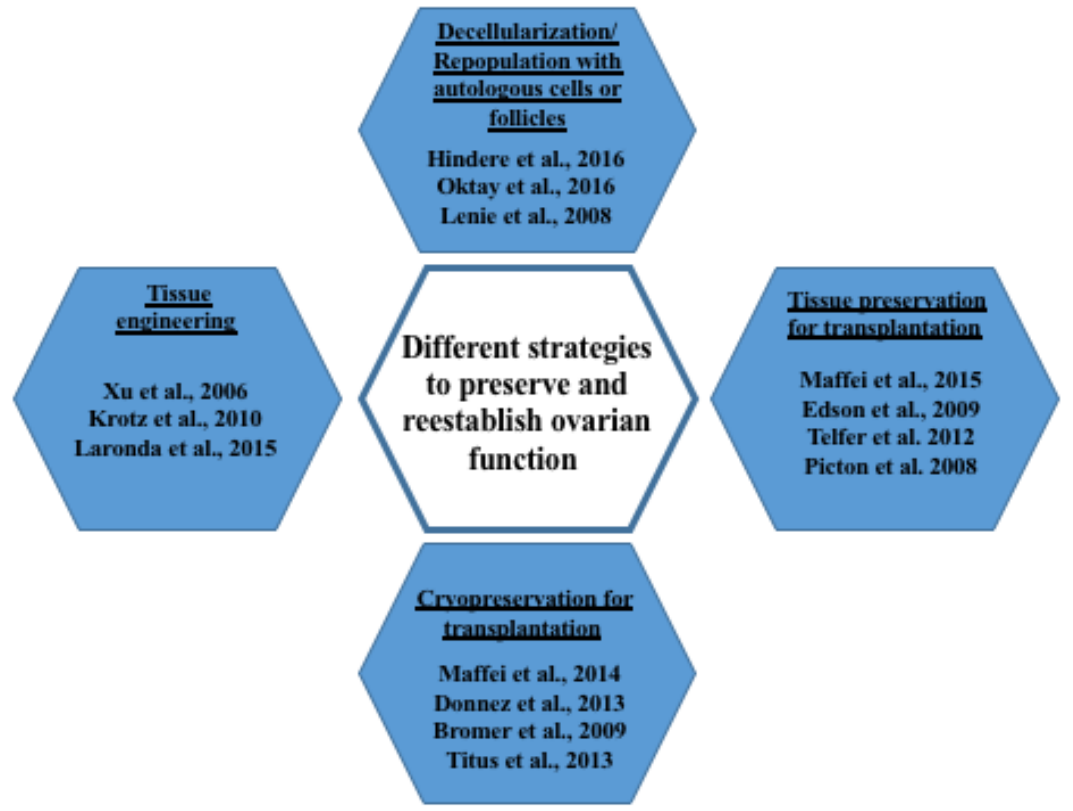

Figure 1. Different strategies presently used to restore ovarian function.

\section{Ovarian tissue preservation for transplantion strategies}

Transplantation of cryopreserved ovarian follicles has been a standard clinical practice for a while, with relatively good success. This can be a vital strategy for young cancer patients who have to undergo chemotherapy and/or pelvic radiotherapy and typically requires the transplant of autologous ovaries to areas outside the irradiation area or maintained ex vivo until completion of chemotherapy. At remission, the preserved tissue can be returned to the donor. The treatment utilizes autologous ovaries so there are no issues related to rejection or need for immunosuppression. It can be greatly advantageous and indeed shown to allow recovery of fertility after total body irradiation and chemotherapy (Larsen et al., 2000; Rodriguez-Walleberg et al., 2015). However, preservation of functional activity during ex vivo maintenance has been a challenge tackled by the research of the last year, considering that the ovary is a complex organ that requires an intricate and dynamic three-dimensional structure in order to sustain follicle development and oocyte maturation (Picton et al., 2008; Edson et al., 2009; Telfer and McLaughlin, 2012). Numerous efforts have been dedicated to the design of culture protocols that allow optimal results. Nevertheless, with the exception of the mice, progress has been limited because of the longer period required for natural folliculogenesis, in larger species and for the related need for extended culture time to generate fully grown follicles and oocytes. A further problem affecting the functional ability of preserved tissue is the difficulty to mimic in vitro the complex relationship that takes place physiologically with the surrounding stroma and with the other ovarian structures. A possible option is the perfusion of the whole ovary, so that its entire architecture can be maintained intact and the supply of hormones and other regulatory factors through the blood circulation can be mimicked by a pump. This approach, which was first demonstrated by early experiments in the mouse, rat and rabbit, was more recently applied to larger species has been as a tool to test the viability and function of cryopreserved whole ovaries. We recently developed a perfusion system that allowed us to maintain functional whole sheep ovaries for up to 4 days. The data generated in those experiments demonstrated that the protocol was able to preserve functional activity of both fresh and cryopreserved ovaries, with identical hormonal secretion levels and comparable estradiol and progesterone production. These results were supported by morphological evidence that showed very good preservation of structural features in fresh and frozen tissue. Altogether, the evidence obtained supported the concept that an extended ex vivo culture of whole ovaries in larger species is possible even after cryopreservation and could lead to the retrieval of competent oocytes in the near future. Furthermore, this culture system could be applied to complement current protocols of cryopreservation of ovarian tissue for preserving fertility of human patients that survive cancer and provides an in vitro relevant model for physiological and toxicological studies.

\section{Cryopreservation of ovarian tissue: conventional and directional freezing}

This approach can be achieved through the excision and banking of whole organs or of fragments of the cortical region. It is however interesting to note that, whereas very low success was reported following the cryopreservation of whole human ovaries, 24 live babies have been obtained after transplantation of frozen-thawed ovarian fragments (Donnez et al., 2013). This clearly suggests that the use of ovarian fragments is the method of choice even if, at present, the average functional life span of these samples ranges from 2 to 5 
years (Bromer and Patrizio, 2009; Donnez et al., 2013). In theory, the cryopreservation of whole ovaries followed by the vascular anastomosis of the ovarian pedicle of the thawed organ should be able to guarantee a better follicular reserve and a longer life span of the transplant. However, the data available do not point in this direction. We recently demonstrated that this is largely due to the damage caused by the freezing procedures applied and showed that both ovarian structure and function were significantly better preserved when selecting an appropriate freezing procedure (Maffei et al., 2014). In particular, we could show that directional freezing was able to significantly improved the integrity of all follicular structure, by maintaining a precise cooling rate and thus preventing the formation of intracellular ice.

Directional freezing was beneficial for both entire ovaries and ovarian fragments and the results obtained clearly indicated a significant improvement versus the use of conventional cryopreservation strategies. We observed a higher cell proliferation rate and a better morphology. Furthermore, when we examined the expression of Heat Shock Proteins (HSPs), which are among the best characterized responses to thermal stress, we detected significantly stimulated, over baseline levels, only in the case of conventional freezing. Even more interestingly, we could observe that DNA damage was significantly lower in directional freezing samples and was always followed by a more intense DNA repair activity with the formation of a Rad51-ssDNA nuclefilament, which is an essential step allowing full DNA double helix integrity re-establishment (Seeber et al., 2013).This is a very important advantage of directional freezing and is particularly relevant for long-term function after thawing, since DNA double strand break repair efficiency has been identified as an important determinant of oocyte aging in women (Titus et al., 2013).

Altogether, we can affirm that directional freezing could represent a promising strategy for the preservation/transplantation of whole ovaries. However, the persistent technical difficulties linked to the surgical procedures required for re-transplantation is likely to still limit the use of whole organs in clinical settings. On the other hand the increasing concerns related to the danger of transferring malignant cells with transplanted frozen-thawed ovarian tissue may lead to a re-evaluation of the technique in terms of using whole ovaries as a source of high quality isolated follicles to be grown in artificial ovaries (Vanacker et al., 2012; Fig. 2).
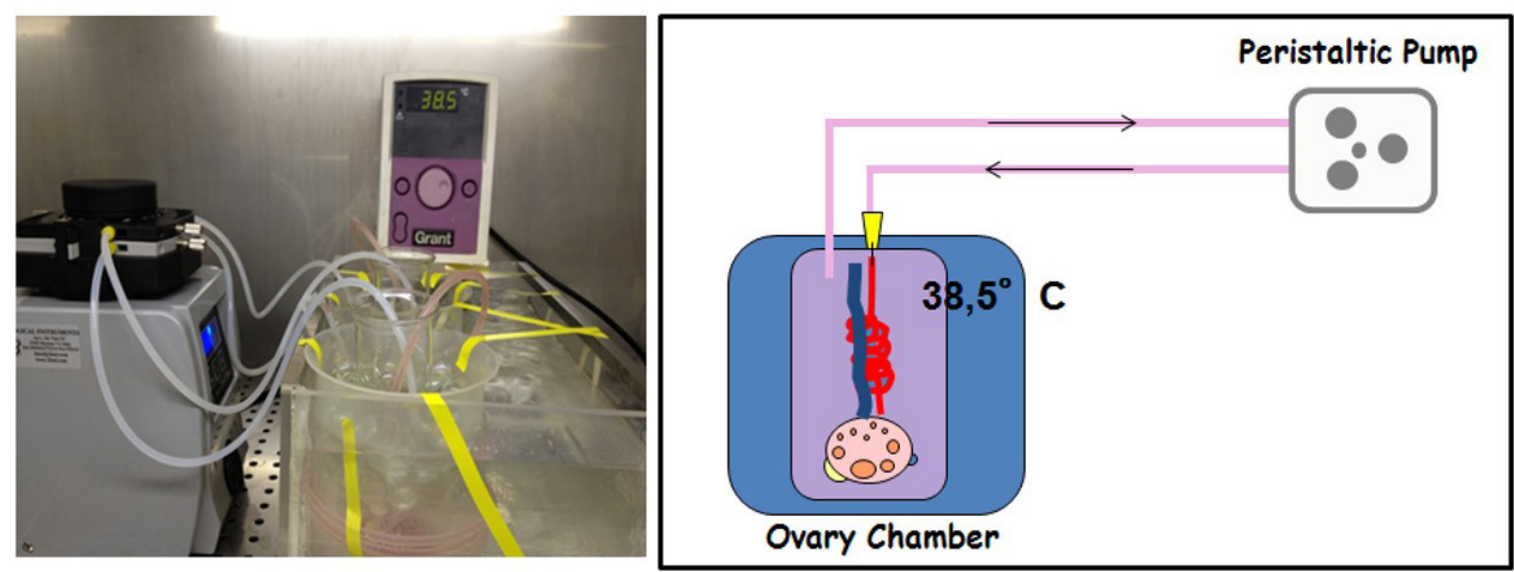

Figure 2. Perfusion systems allow to maintain functional whole sheep ovaries for several days, preserving functional activity of both fresh and cryopreserved organs, with identical hormonal secretion levels and comparable estradiol and progesterone production.

\section{Tissue engineering the ovary}

Great efforts have been addressed lately in order to avoid reintroducing the disease in patients. In particular, scientists have developed tissue engineered oocyte maturation systems to activate oocytes isolated from cryopreserved ovarian tissues. Pangaset al. developed a $3 \mathrm{D}$ in vitro culture system with alginate beads that was used to grow individual granulosa celloocyte complexes (GOCs) and provided complexes with spatial arrangement (Xu et al., 2006). The alginatebased system was shown to yield far more oocytes than conventional IVF, as the majority of mammalian oocytes are contained in immature and quiescent follicles (Xu et al., 2006). Moreover, Xu et al. (2006) isolated immature follicles, cultured them in alginate droplet, fertilized in vitro and transferred to pseudopregnant female mice. They were able to generate viable offspring, of both female and male gender and fertile (Xu et al., 2006).

Very promising results were also obtained using agarose, which is another polysaccharide-based biomaterial, widely used in tissue engineering, and that was used as a matrix for in vitro maturation of oocytes (Che-Ying et al., 2016). The three follicular cell typestheca, granulosa, and oocytes were homed in agarose to create a $3 \mathrm{D}$ artificial human ovary that was shown to allow successful maturation of early antral follicle $(<10$ $\mathrm{mm}$ ) oocytes into metaphase II oocytes (Che-Ying et al., 2016). Although very encouraging, these approaches that used polysaccharide biomaterials alone, provided a suboptimal environment because they lacked 
ECM proteins that are essential to the phenotypes of mammalian cells (Huet et al., 2001). Because of this, other studies utilizing biomaterials added with ECM components such as RGD, collagen (type I and IV), and/or fibronectin, demonstrated improved growth, differentiation, and meiotic competence of the oocyte (Kreeger et al., 2003; Kreeger et al., 2006; Laronda et al., 2015). Further advantages were recently derived, adopting 3D bio-printing techniques that allows the seeding and the culture of different cell types recreating a layer-by-layer environment that builds a cyto/histological architecture similar to the original tissue (Irvine et al., 2015). The creation of the supports printed with compatible materials that can then be repopulated with the specific cell subpopulations, or the direct printing of a cells embedded matrix are the two possible options presently available.

In the field of human and veterinary reproduction, the use of $3 \mathrm{D}$ printers is useful and necessary for the creation of ovarian prostheses or part of tissues supporting ovarian cells (Hinderer et al.,
2016). In the near future bio-printing applied to reproductive medicine will be table to create a multilayered functional culture, composed by different tissues and stratifications and develop a functional ovary with anatomical and physiological characteristic of the native organ (Shea et al., 2014). This may result in terrific advantages, restoring hormone and fertility function in onco-fertility patients. A very exciting report by Laronda et al. (2015) described the generation of a 3D-printed mouse model of ovarian follicles on a hydrogel support, with a compatible cyto-architecture like the original organ (Laronda et al., 2015). The follicle-seeded scaffolds became highly vascularized and was able to fully restore ovarian function when implanted in surgically sterilized mice. Moreover, pups were born through natural mating and thrive through maternal lactation. These findings indicate the possibility to create an in vivo functional ovarian implant, designed with 3D printing, and confirm tissue engineering as a tremendously promising strategy for regenerative reproductive biology (Fig. 3).

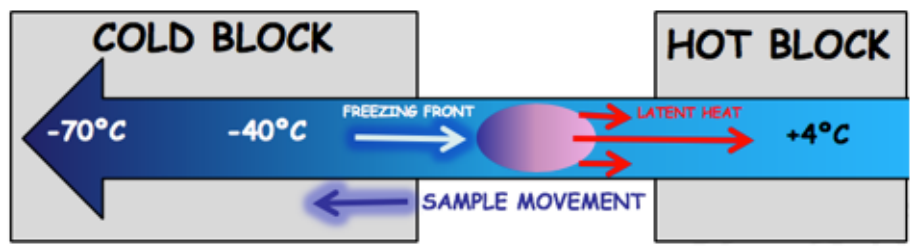

SAMPLE MOVEMENT:

$0.01 \mathrm{~mm} / \mathrm{s}, 0.3^{\circ} \mathrm{C} / \mathrm{min}$

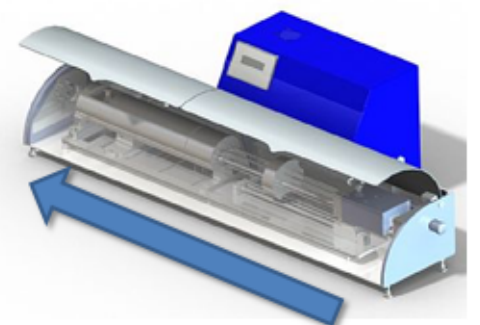

Figure 3. In directional freezing, the freezing rate is determined by the combination of temperature gradient and speed of the sample along the track. If the velocity of the sample is slower than the speed at which the heat is removed from the center of the sample towards its periphery, heat transfer is quickly removed in the direction opposite to that of the sample movement. All this results in a uniform cooling rate throughout the sample. The 3 thermal blocks are generally set at $4^{\circ},-10^{\circ}$ and $-70^{\circ} \mathrm{C}$ respectively, thereby imposing a temperature gradient around the tubes. Freezing tubes are pushed lengthwise, along the thermal gradient, and the speed is set at $0.01 \mathrm{~mm} / \mathrm{s}$, resulting in a cooling rate of $0.3^{\circ} \mathrm{C} / \mathrm{min}$ down to $-70^{\circ} \mathrm{C}$. At the end of the procedure, samples are plunged into liquid nitrogen.

\section{Decellularization/repopulation of ovary with autologous cells or follicles}

The field of regenerative medicine and tissue engineering has been developing for decades different techniques that promote and allow the proliferation of cells belonging to different tissues. One of these is the methodology of organ decellularization, a real removal of cells from parenchyma and non-parenchyma organs. The consequence of this method is to create a support and a scaffold for a subsequent homing of variegated cell types (Oktay et al., 2000; Shea et al., 2014; Hinderer et al., 2016). During the static or dynamic decellularization processes, the portion of tissue known as ECM is produced. There are several reports describing methodologies and applications to obtain a native and pure ECM without creating structural damage, thus obtaining an unusable scaffold. Remarkable capacity to promote cellular and tissues regeneration in various organs such as kidney, heart, lung and liver have been studied and described but few studies developed and showed this particular technique 
and its important role in primordial follicle growth and survival (Oktem and Oktay, 2007, Tapias and ott, 2014; Pati et al., 2014; Quian et al., 2015; Oktay et al., 2016). Indeed, the new field of ovary and uterus decellularization is developing continuously, filling the gap between the laboratory practice and the reproductive organ and cell needs in clinical transplantation. Moreover, the developed capacity to create anad hoc organ or 3D cell culture using the decellularization technique is evolving the field of Precise Medicine applications. For this reason the main scope of the decellularization is the ability to use the own patient cells and genetic kit to avoid and solve the obstacle of the organ rejection or data translation from in vitro to in vivo applications (Gilbert et al., 2006; Keane et al., 2015). The main point is to create ECMbased biomaterials, comprising non water-soluble structural proteins as collagens, laminins, elastin or proteoglycans (PGs), in association with important hormones and growth factors, used as stimulating compound, to better increase cell proliferation and organoid growth (Hinderer and Schenke-Layland $\mathrm{K}$. 2013). To obtain the first native ECM material, different physical treatments such as agitation, sonication, mechanical pressure, or freeze-thawing procedures, let to disrupt cell membrane, in combination with washing solutions as deionized water and various ionic or nonionic detergents (Andrée et al., 2014). Enzymatic treatment using trypsin, dispase, esterases or nucleases has also been employed to remove cells from tissues, becoming a part of the standard protocol used in daily lab. The ECM obtained with the different approaches described above, is utilized as a 3D fibrous and porous scaffold, with original nets and vasculature preserved. Needless to say, all the procedures finalized to remove the original cells from the organ has to avoid the deterioration of the matrix components (Hinderer et al., 2013). Most of the reports available, utilize ultrastructural analyses to evaluate the impact of the decellularization method chosen in damaging the crucial structural tissue elements such as elastic fiber (Lenie et al., 2008). For instance, PGs and glycosaminoglycans, which connect cells to collagen bundles or elastic fibers and induce a variety of cellular responses, must be fully preserved in decellularized tissues (Paulose et al., 2012), to allow for coordinated growth of the multiple cellular compartments of the follicle-the oocyte, granulosa cells, and theca cells-in vitro. The techniques used in our laboratory are selected based on the idea to cause the lesser mechanical and chemical stress for the biomatrix, but, at the same time, generate efficiently decellularized ECM. These involve the use of gradual temperature shock following a chemical treatment with Triton $\mathrm{X}$ and sodium dodecyl sulfate (SDS), utilizing a peristaltic pump with a low flow, low pressure and long perfusion time (Ghiringhelli et al., 2017). This experimental strategy allows for the obtainment of a well-preserved bio-scaffold that may constitute a promising environment to support the dynamic ovarian environment and the optimal niche for follicle maturation. The future prospective of ovary transplantation could be supported and implemented by all type of decellularized ECM materials, giving the chance to recreate new functional ovary and, avoiding the difficult obstacle of the short organ donors and the rejection during ovarian transplantation (Fig. 4).

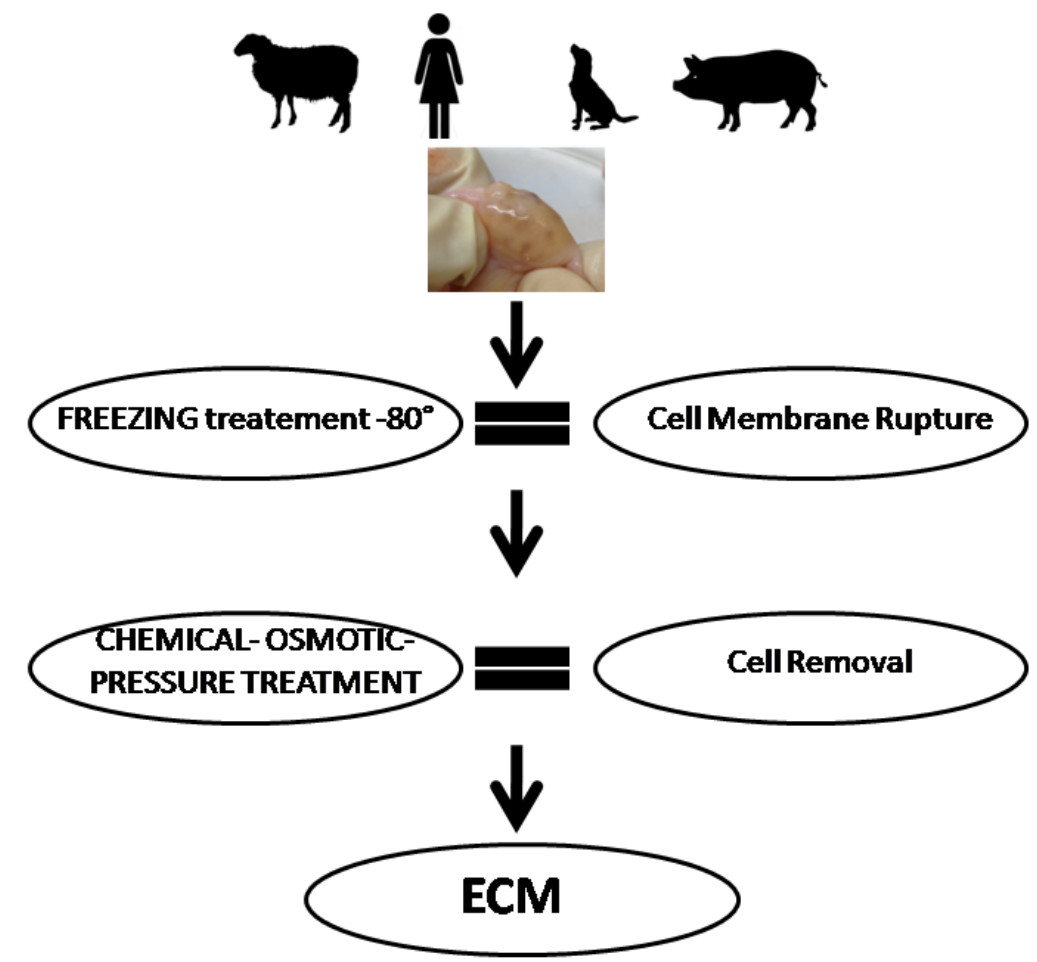

Figure 4. Schematic study plan to obtain a decellularized extracellular matrix from ovary. From the fresh ovary to the ECM material, the tissue is passed through the thermic treatment to obtain cell rupture. Effective cell removal is achieved thanks to the use of different ionic and non-ionic detergents, such as Triton X100, SDS and Deoxycolic acid and combining them to basic compounds such as ammonium hydroxide. 


\section{Conclusions}

The ovarian environment is complex and dynamic, it is modulated by cyclic changes in endocrine factors, both deriving from the systemic circulation as well as from local signals. In addition, the function and properties exerted by the ECM further contribute to the interactions with the follicle and its somatic cells 14,15 Different bioengineering strategies have been presently adopted and shown to have great potential in order to preserve, maintain and or recreate the ovarian milieau. Transplantation of fresh or cryopreserved organs/fragments that re-establish ovarian function have been recently paired with the use of different and complex combination of cells, biomaterials and factors that stimulate local ability to regenerate the reproductive organ and methods that employ decellularization/repopulation of ovary with autologous cells or follicles. All these different options represent interesting, although still experimental alternatives to preserve or improve female fertility and are very promising strategies of regenerative reproductive medicine.

\section{Acknowledgments}

This work was funded by Carraresi Foundation. The Authors are members of the COST Actions CA16119 and CM1406.

\section{References}

Anderson RA, Wallace WH. 2011. Fertility preservation in girls and young women. Clin Endocrinol (Oxf), 75:409-419.

Andrée B, Bela K, Horvath T, Lux M, Ramm R, Venturini L, Ciubotaru A, Zweigerdt R, Haverich A, Hilfiker A. 2014. Successful re-endothelialization of a perfusable biological vascularized matrix (BioVaM) for the generation of 3D artificial cardiac tissue. Basic Res Cardiol, 109:1-13.

Bromer JG, Patrizio P. 2009. Fertility preservation: the rationale for cryopreservation of the whole ovary. Semin Reprod Med, 27:465-471.

Che-Ying K, Avinash E, Jesse KP, Kelly RR, Helim AE, Rohan F, John PF, Peter CW. 2016. Development of a 3D printed, bioengineered placenta model to evaluate the role of trophoblast migration in preeclampsia. ACS Biomater Sci Eng, 2(10):1817-1826.

Chung K, Donnez J, Ginsburg E, Meirow D. 2013. Emergency IVF versus ovarian tissue cryopreservation: decision making in fertility preservation for female cancer patients. Fertil Steril, 99:1534-1542.

Dolmans MM, Marinescu C, Saussoy P, Van Langendonckt A, Amorim C, Donnez J. 2010 Reimplantation of cryopreserved ovarian tissue from patients with acute lymphoblastic leukemia is potentially unsafe. Blood, 116:2908-2914.

Donnez J, Dolmans MM, Pellicer A, Diaz-Garcia C, Sanchez Serrano M, Schmidt KT, Ernst E, Luyckx V, Andersen CY. 2013. Restoration of ovarian activity and pregnancy after transplantation of cryopreserved ovarian tissue: a review of 60 cases of reimplantation. Fertil Steril, 99:1503-1513.

Edson MA, Nagaraja AK, Matzuk MM. 2009. The mammalian ovary from genesis to revelation. Endocr Rev, 30: 624-712.

Ghiringhelli M, Zenobi A, Brizzola S, Gandolfi F, Bontempo V, Rossi S, Brevini TAL, Acocella F. 2017. Simple and quick method to obtain a decellularized, fuctional liver bioscaffold. Methods Mol Bio,

Gilbert TW, Sellaro TL, Badylak SF. 2006. Decellularization of tissues and organs. Bio-materials, 27:3675-3683.

Greve T, Clasen-Linde E, Andersen MT, Andersen MK, Sorensen SD, Rosendahl M, Ralfkiaer E, Andersen CY. 2012. Cryopreserved ovarian cortex from patients with leukemia in complete remission contains no apparent viable malignant cells. Blood, 120:4311-4316.

Hewlett M, Mahalingaiah S. 2015. Update on primary ovarian insufficiency. Curr Opin Endocrinol Diab Obes, 22(6):483-489.

Hinderer S, Schenke-Layland K. 2013. Tracheal tissue engineering: building on a strong foundation, Expert Rev Med Devices, 10:33-35.

Hinderer S, Layland SL, Schenke-Layland K. 2016. ECM and ECM-like materials - Biomaterials for applications in regenerative medicine and cancer therapy. Adv Drug Deliv Rev, 97:260-269

Huet C, Pisselet C, Mandon-Pepin B, Monget P, Monniaux D. 2001. Extracellular matrix regulates ovine granulosa cell survival, proliferation and steroidogenesis: relationships between cell shape and function. J Endocrinol, 169(2):347-60.

Irvine SA, Agrawal A., Lee BH, Chua HY, Low KY, Lau BC, Machulf M, Venkatraman S. 2015. Printing cell-laden gelatin constructs by free-form fabrication and enzymatic protein crosslinking. Biomed Microdevices, 17:16-18.

Keane TJ, Swinehart IT, Badylak SF. 2015. Methods of tissue decellularization used for preparation of biologic scaffolds and in vivo relevance. Methods, 84:25-34

Kreeger PK, Woodruff TK, Shea LD. 2003. Murine granulosa cell morphology and function are regulated by a synthetic Arg-Gly-Asp matrix. Mol Cell Endocrinol, 205(1-2):1-10.

Kreeger PK, Deck JW, Woodruff TK, Shea LD. 2006.The in vitro regulation of ovarian follicle development using alginate-extracellular matrix gels. Biomaterials, 27(5):714-723.

Krotz SP, Robins JC, Ferruccio TM, Moore R, Steinhoff MM, Morgan JR, Carson S. 2010. In vitro maturation of oocytes via the prefabricated selfassembled artificial human ovary. $J$ Assist Reprod Genet, 27(12):743-750.

Laronda MM, Jakus AE, Whelan KA, Wertheim JA, Shah RN, Woodruff TK. 2015. Initiation of puberty in mice following decellularized ovary transplant. Biomaterials, 50:20-29.

Larsen E, Loft A, Holm K, Muller J, Brocks V, Andersen AN. 2000. Oocyte donation in women cured of cancer with bone marrow transplantation including 
total body irradiation in adolescence. Hum Reprod, 15(7):1505-1508.

Lenie S, Cortvrindt R, Eichenlaub-Ritter U, Smitz J. 2008 Continuous exposure to bisphenol A during in vitro follicular development induces meiotic abnormalities. Mutat Res, 651:71-81.

Maffei S, Pennarossa G, Brevini TA, Arav A, Gandolfi F. 2014. Beneficial effect of directional freezing on in vitro viability of cryopreserved sheep whole ovaries and ovarian cortical slices. Hum Reprud, 29(1):114-124.

Maffei S, Galeati G, Pennarossa G, Brevini TAL, Gandolfi F. 2015. Extended ex vivo culture of fresh and cryopreserved whole sheep ovaries. Reproduction, Fertility and Development, 28(12):1893-1903.

Meirow D, Hardan I, Dor J, Fridman E, Elizur S, Ra'anani H, Slyusarevsky E, Amariglio N, Schiff E, Rechavi G, Nagler A, Ben Yehuda D. 2008. Searching for evidence of disease and malignant cell contamination in ovarian tissue stored from hematologic cancer patients. Hum Reprod, 23:1007-1013.

Oktay K, Karlikaya G, Akman O, Ojakian GK, Oktay M. 2000. Interaction of extracellular matrix and activin-A in the initiation of follicle growth in the mouse ovary. Biol Reprod, 63:457-461.

Oktay K, Bedoschi G, Pacheco F, Turan V, Emirdar V. 2016. First pregnancies, live birth, and in vitro fertilization outcomes after transplantation of frozenbanked ovarian tissue with a human extracellular matrix scaffold using robot-assisted minimally invasive surgery. Am J Obstet Gynecol, 214:94. e1-94.e9.

Oktem O, Oktay K. 2007. The role of extra-cellular matrix and activin-A in in vitro growth and survival of murine preantral follicles. Reprod Sci, 14:358-366.

Pangas SA, Saudye H, Shea LD, Woodruff TK. 2003. Novel approach for the three-dimensional culture of granulosa cell-oocyte complexes. Tissue Eng, 9(5):1013-21.

Pati F, Jang J, Ha DH, Kim SW, Rhie JW, Shim JH, Kim DH, Cho DW. 2014. Printing three-dimensional tissue analogues with decellularized extracellular matrix bioink, Nat Commun, 5:3935.

Paulose T, Tannenbaum LV, Borgeest C, Flaws JA. 2012. Methoxychlor-induced ovarian follicle toxicity in mice: dose and exposure duration-dependent effects. Birth Defects Res B Dev Reprod Toxicol, 2012; 95:219224.

Picton HM, Harris SE, Muruvi W, Chambers EL. 2008. The in vitro growth and maturation of follicles. Reproduction, 136:703-715.
Qin YY, Jiao X, Simpson JL, Chen ZJ. 2015. Genetics of primary ovarian insufficiency: new developments and opportunities. Hum Reprod Update, 21(6):787-808.

Rodriguez-Wallberg K, Karlstrom PO, Rezapour M, Castellanos E, Hreinsson J, Rasmussen, Sheikhi M, Ouvrier B, Bozoky B, Olofsson JI, Lundgvist M, Hovatta O. 2015. Full-term newborn after repeated ovarian tissue transplants in a patient treated for Ewing sarcoma by sterilizing pelvic irradiation and chemotherapy. Acta Obstet Gynecol Scand, 94(3):324-8. Sadri-Ardekani H, Atala A. 2015. Regenerative medicine for the treatment of reproductive system disorders: current and potential options. Adv Drug Deliv Rev, 82:45-158.

Seeber A, Hauer $M$ and Gasser SM. 2013. Nucleosome remodelers in double-strand break repair. Curr Opin Genet Dev, 23:174-184.

Shea LD, Woodruff TK, Shikanov A. 2014. Bioengineering the ovarian follicle microenvironment. Annu. Rev. Biomed. Eng. 16:29-52.

Tapias LF, Ott HC. 2014. Decellularized scaffolds as a platform for bioengineered organs. Curr Opin Organ Transplant, 19:145-152.

Telfer EE, McLaughlin M. 2012. Strategies to support human oocyte development in vitro. Int $J$ Dev Biol, 56:901-907.

Titus S, Li F, Stobezki R, Akula K, Unsal E, Jeong K, Dickler M, Robson M, Moy F, Goswami S, Oktay K. 2013. Impairment of BRCA1-related DNA doublestrand break repair leads to ovarian aging in mice and humans. Sci Transl Med, 5(172):172ra21.

Vanacker J, Luyckx V, Dolmans MM, Des Rieux A, Jaeger J, Van Langendonckt A, Donnez J, Amorim CA. 2012. Transplantation of an alginate-matrigel matrix containing isolated ovarian cells: first step in developing a biodegradable scaffold to transplant isolated preantral follicles and ovarian cells. Biomaterials, 33:6079-6085.

Wallace WH, Anderson RA and Irvine DS. 2005. Fertility preservation for young patients with cancer: who is at risk and what can be offered? Lancet Oncol, 6:209-218.

Xu M, Kreeger PK, Shea LD, Woodruff TK. 2006. Tissue-engineered follicles produce live, fertile offspring. Tissue Eng, 9(5):1013-1021.

Yalcinkaya T, Sittadjody S, Opara E. 2014. Scientific principles of regenerative medicine and their application in the female reproductive system. Maturitas, 77(1):1219 